\title{
Eye-Movement-Dependent Loss in Vision and Its Time Course During Vergence
}

\author{
Karen A. Manning \\ W. S. Hunter Laboratory of Psychology, Brown University, Providence, Rhode Island 02917
}

The attenuation of vision that has long been known to accompany saccadic eye movement has a significant component that is not attributable to visual masking or image smear, and this suppression of vision is now associated with nonsaccadic movement. The purpose of the present experiment was to determine the time course of visual suppression during a vergence eye movement.

Suppression was evaluated psychophysically in human observers by measuring their loss of sensitivity to a brief, full-field decrement of light during $6^{\circ}-9^{\circ}$ convergence. Vergence-related suppression was similar in total duration and amplitude to saccadic and blink-related suppression. Since these other forms of oculomotor activity are vastly different in speed and total duration, it is unlikely that suppression results directly from the activity itself. Instead, these results support the hypothesis that a common, more centrally originating, suppression of vision occurs during eye movements, including saccades, eyeblinks, and vergence. Thus, while vision during eye movements can often be reduced through masking and smearing effects, the movementdependent visual suppression measured in these experiments is a more generally occurring event.

The question of what happens to vision during movement of the eyes has long been an intriguing and controversial issue. Some investigators have thought that vision during eye movements such as saccades remains essentially unaffected (MacKay, 1973; Woodworth, 1906, 1938), while others have claimed almost the opposite, that an extensive or complete loss of vision accompanies each eye movement so that continuous, clear vision is an illusion (Dodge, 1900, 1905; Holt, 1903). The predominant finding of most studies on vision during saccades (see Matin, 1974, and Volkmann, 1976, for reviews) is that vision is, in fact, impaired.

However, diminished vision during saccades must arise through not one, but several processes. Visual masking, a reduction in sensitivity to a visual stimulus due to the close proximity in time or space of another visual stimulus, can occur whenever there is a rapid change in the location of contours in the visual scene (Alpern, 1953; Breitmeyer and Ganz, 1976; Lefton, 1972; Weisstein, 1972). Also, the image of an object may, in effect, be smeared across the retina over the course of a rapid eye movement, making it harder to be seen (Matin, 1974; Mitrani et al., 1970). The combination of these 2 visual

\footnotetext{
Received Oct. 15, 1985; revised Dec. 23, 1985; accepted Dec. 30, 1985
}

This work was supported by NIH Grant EY03169 to Lorrin A. Riggs. I am deeply grateful to Dr. Riggs for his contributions to every aspect of this research. I thank R. K. Moore for his technical assistance and advice and C. Evinger for his critical comments on an earlier version of this manuscript. Submitted in partial fulfillment of the requirements for the degree of Doctor of Philosophy in Psychology at Brown University.

Correspondence should be addressed to Karen A. Manning, Department of Neurobiology and Behavior, State University of New York at Stony Brook, Stony Brook, New York 11794.

Copyright (C) 1986 Society for Neuroscience $0270-6474 / 86 / 071976-07 \$ 02.00 / 0$ effects may greatly reduce vision during rapid saccadic eye movements when viewing conditions are well illuminated and highly contoured (Brooks and Fuchs, 1975; Campbell and Wurtz, 1978; MacKay, 1973). However, neither effect requires movement of the eyes.

In addition, vision during saccades is diminished through an inhibition of vision that is associated with making the movement itself. When vision during a saccade is tested under conditions that minimize or eliminate the effects of visual masking or smear, a substantial loss in visual sensitivity occurs that can last $300-400 \mathrm{msec}$ and includes a loss in contrast sensitivity (Riggs and Manning, 1982; Riggs et al., 1974; Volkmann, 1962; Volkmann et al., 1968, 1978a). When stimuli are presented near the start of the saccade, the loss in sensitivity is near maximum and measures $0.5-1.0 \mathrm{log}$ unit. Such visual suppression appears to be produced through a centrally arising process associated with initiation of the eye movement. Helmholtz (1954), and more recent authors, demonstrated that during naturally emitted eye movement, the brain acts in some manner to compensate for sensory effects of the movement. An efferent signal in the brain (Sperry, 1950; von Holst, 1954) such as a feed-forward corollary signal to the command to move the extraocular muscles has been considered the most likely source of neural signals that affect vision during eye movements (Armington, 1981; Collewijn, 1969; Duffy and Lombroso, 1968; Gregory, 1966; Gross et al., 1967; Latour, 1962; Riggs et al., 1974, 1982; Volkmann, 1962, 1976; Volkmann et al., 1978a, b; White et al., 1984; Zuber and Stark, 1966; Zuber et al., 1964, 1966).

Saccades are not the only eye-related movements that are accompanied by movement dependent visual suppression. All types of blinks, whether voluntary (Armington, 1981; Riggs et al., 1981; Volkmann et al., 1979, 1980; Wibbenmeyer et al., 1983), reflex (Manning et al., 1983b), or spontaneous (Manning et al., 1983a), and changes in lid position (Volkmann et al., 1982) result in suppression. Now, a preliminary study indicates that suppression is associated with vergence eye movements (Manning and Riggs, 1984).

The similarity of visual suppression measured during these different oculomotor movements, when the effects of masking and smear are minimized or eliminated, suggests a common neural mechanism. Yet vergence eye movements are different in practically every respect from saccadic eye movements. Convergence and divergence are extremely slow, smooth, disjunctive movements of the eyes that allow us to focus between near and distant objects and differ from saccades in their dynamics and in the neural commands that generate them (Bahill and Stark, 1979; Keller and Robinson, 1972; Mays, 1983, 1984; Mays and Porter, 1984). A vergence movement usually requires much more time to be completed than does a saccade of the same amplitude, and during such a vergence movement, the peak velocity and acceleration attained by the eyeball are considerably lower than those measured during a saccade. Masking effects, like those that can arise during saccides, are unlikely to 
arisc during vergence. Any shcaring strains within the cyc during saccadic eye rotation that could conceivably degrade vision (Richards, 1968, 1969; also see Grehn et al., 1984) should greatly exceed those that could occur during vergence.

The present experiments examined in detail the suppression of vision during vergence under conditions that minimized, or eliminated, visual masking and smear. If visual suppression results from specific inhibitory effects of eye rotation or its shearing effect, then the duration of the loss in sensitivity during long, smooth vergence should be significantly longer than that measured during quicker, high-velocity saccades and the time course of the change in sensitivity should differ from that measured during saccades. Alternatively, if visual suppression represents a more general, centrally controlled process, then the pattern of change in sensitivity might well be similar regardless of the type of eye movement with which it is associated. The purpose of the experiment was therefore to examine the time course of visual suppression that accompanies a convergent eye movement and to compare this result with that obtained previously for saccades.

\section{Materials and Methods}

\section{Viewing conditions}

Experiments were conducted in a $60-\mathrm{cm}$-diameter Ganzfeld (Riggs et al., 1981) providing approximately uniform illumination of the entire visual field. Subjects rested their chin in a clear, Plexiglas chin rest that kept the head centered in the globe and viewed the smooth, white (Nextel Velvet coating, 101-A10, 3M Co.) inner surface of the sphere. Three pairs of fluorescent lamps (6 W, ED3, Electronic Developments) positioned symmetrically behind the subject's head and outside the field of view illuminated the Ganzfeld. Each pair of lamps was screened with an aluminum lattice that maintained the uniform spectral and spatial distribution of light in the Ganzfeld and a photopic luminance level of 24-26 foot-Lamberts (LiteMate, Photo Research). Lamps with fast rise and fall times (Mollon and Polden, 1978) were operated with DC current by a regulated tachistoscopic power supply (Iconix).

The visual test stimulus was a $20 \mathrm{msec}$ full-field decrement $(\Delta I)$ in the level of Ganzfeld illumination $(I)$ produced by a circuit that delivered a square-wave, negative pulse in the current to the fluorescent lamps. Fixed-step attenuators produced equal steps of $\log (\Delta I / I)$ calibrated by the use of a fast photocell. These steps ranged from clearly visible to imperceptible decrements. Testing vision in the featureless Ganzfeld with these extremely brief, diffuse flashes minimizes visual masking and smear and provides the same retinal stimulus to the stationary or moving eye (Riggs et al., 1982).

Two tiny targets guided the subject's symmetrical vergence movements (Fig. 1). The far target was a yellow spot of light that was produced by focusing the tungsten filament image of a miniature projector onto the Ganzfeld surface. The projector itself was outside the subject's field of view. The near target was an ink spot on a microscope coverslip held upright in the center of the Ganzfeld on a clear Plexiglas rod roughly $15 \mathrm{~cm}$ away from the subject. The far and near targets subtended 17 and $23 \mathrm{~min}$ of visual angle, respectively, and were centered in the field of view along the horizontal plane of the eyes. The different colors helped the subject to distinguish the near from the far target. When the subject converged his eyes to binocularly fixate the near spot, the far target appeared as 2 yellow spots located symmetrically to the right and left of fixation. When the subject diverged his eyes to fixate the far (yellow) target, the near (black) target appeared as double. By maintaining the symmetrical arrangement of the spots that were perceived during target fixation, subjects aligned themselves so that symmetrical vergence movements ensued as they looked from one target to the other. A projected scale within the Ganzfeld was illuminated to check target position during each session. Targets were positioned to elicit a relatively large, yet easily executed, symmetrical vergence movement. A small translational movement that may accompany vergence (Enright, 1984) was ignored in calculating the angle of rotation of each eye. For subjects J.P.K., K.A.M., and S.B.S., respectively, the angle of rotation was $8.7^{\circ}$, $6.2^{\circ}$, and $7.6^{\circ}$. The average corresponding value in prism diopters, for the near and far targets, respectively, equaled $43.6^{\Delta}(\mathrm{SD}=4.8)$ and $16.4^{\Delta}$ $(\mathrm{SD}=1.2)$.

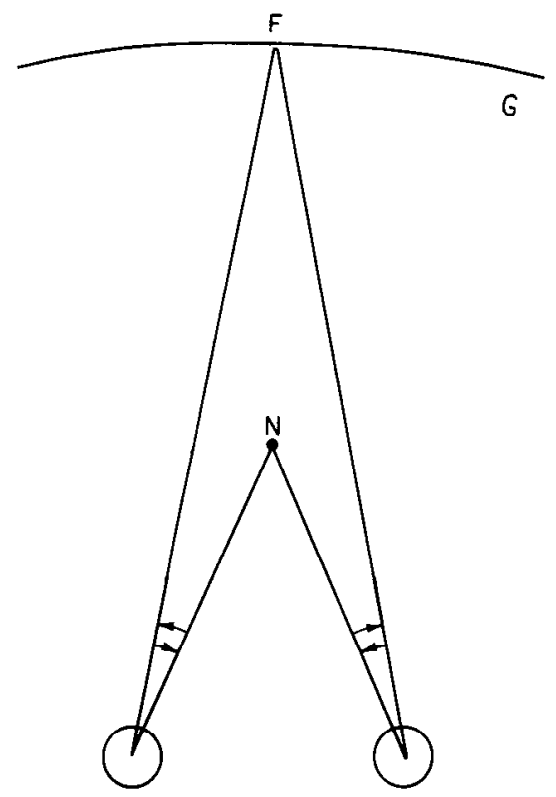

Figure 1. Diagram of vergence movement (not drawn to scale). Subjects executed symmetrical convergence or divergence in the Ganzfeld $(G)$ by changing fixation between the near $(N)$ and far $(F)$ targets. The angle of rotation of the eyes is indicated by the arrows.

\section{Electro-oculogram}

The electro-oculogram (EOG) was used to monitor vergence movements reliably and to activate a trigger circuit for measuring the time between the onset of the eye movement and the presentation of the visual stimulus. Electrodes attached near the outer canthus of each eye were connected together and provided one of the active input leads to a differential amplifier (model 113, Princeton Applied Research). A third electrode placed on the bridge of the nose was the second input to the differential amplifier. The amplified and filtered EOG signal (gain = $100 ; 3 \mathrm{~dB}$ corner frequencies, 0.03 and $10 \mathrm{~Hz}$ ) was monitored on a storage oscilloscope. With this electrode arrangement, convergence and divergence produced deflections of the EOG in opposite directions. In preliminary trials, EOGs were obtained from each subject by examining the signals from each eye separately to make sure that vergence movements were not contaminated by saccades.

Photography was used to calibrate the actual movement of the eye with the EOG. At the beginning of the experiment the subject's right eye was photographed and the EOG was recorded while he executed convergence and divergence movements in the brightly illuminated (24 foot-Lamberts) Ganzfeld. A periscope was constructed from a mirror and lens system and positioned to reflect the enlarged image of the eye to a moving picture camera (Reflex 16, Pathe Professional) mounted outside the Ganzfeld. Additional mirrors were arranged outside the Ganzfeld to reflect to the camera the image of the face of the oscilloscope screcn that displayed the EOG. Thus, the eye position and the EOG were recorded on each frame of film. Movies were filmed (4-X Eastman Negative Film) at 24 frames/sec. The data were analyzed by frame-byframe measurement of eye position and excursion of the EOG trace to determine when the EOG trigger detected the start of vergence with respect to the actual initiation of the eye movement. From these data, it was determined that during convergence J.P.K.'s EOG triggered approximately $152 \mathrm{msec}$ after his eye began to move, K.A.M.'s EOG triggered $27 \mathrm{msec}$ after her eye began to move, and S.B.S.'s EOG triggered $156 \mathrm{msec}$ after his eye began to move.

\section{Psychophysical procedure}

A decrease in the subject's visual sensitivity at the time of an eye movement is measured by reference to sensitivity measured when no eye movement occurs under identical conditions of stimulus presentation. Accordingly, visual thresholds were always obtained for both the vergence condition and fixation condition. Thresholds for detection of the decremental stimulus were determined with a bias-free (Pearce and Porter, 1970), 2-alternative forced-choice procedure. At the start of a 


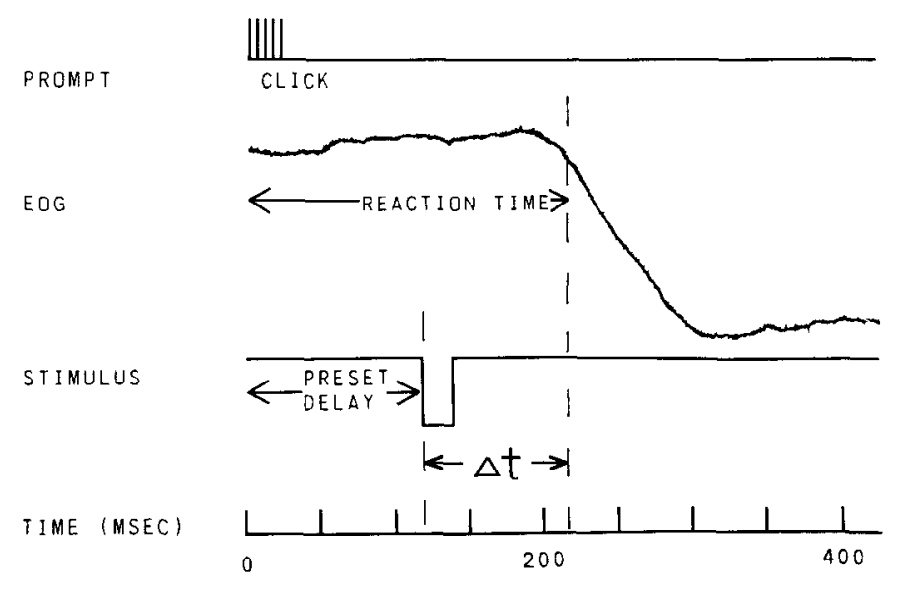

Figure 2. Sequence of experimental events. The auditory prompt signaled the subject to execute a vergence movement. The test stimulus, a $20 \mathrm{msec}$ Ganzfeld light decrement, was presented after a preset time delay of $0-600 \mathrm{msec}$ from the prompt. The interval $\Delta t$ between the detection of vergence by the EOG trigger and the presentation of the test stimulus was recorded after each trial. When stimulus presentation preceded the onset of vergence (as shown here), this time was recorded as a negative value.

convergence trial the subject heard a ready signal that warned him to look at the far target followed by 2 clicks, roughly $3 \mathrm{sec}$ apart. Each click signaled the start of the test interval and prompted the subject to converge. The subject refixated the far target in between clicks. The predetermined value of stimulus decrement was delivered pseudorandomly during either the first or second interval of each trial. From the subject's perspective, the sequence of events that was associated with a decrement was exactly the same as the sequence of events that resulted in no decrement. At the end of each trial the subject's task was to signal whether the stimulus came during the first or the second test interval.

In order to determine the time course of visual suppression, it was nccessary to present the decremental stimulus over a wide range of times, before, during, or after a vergence movement. Figure 2 illustrates the necessary sequence of events. When the click prompted the subject to initiate the vergence movement, a preset liming device delivered the decremental stimulus after a delay of $0-600 \mathrm{msec}$. Once the eye movement and stimulus presentation had occurred, a digital clock measured the time, $\Delta t$, between the presentation of the decremental stimulus and the onset of vergence as marked by a Schmitt trigger in the EOG circuit. The value of $\Delta t(\mathrm{msec})$ was recorded after each trial in which both the decremental stimulus and the vergence movement occurred. Stimuli were presented as early as $300 \mathrm{msec}$ before detection of the EOG and as late as $400 \mathrm{msec}$ after detection of the EOG. The range of time varied somewhat, depending on how quickly the subject initiated convergence. With practice, subjects converged with a fairly constant reaction time, roughly $200 \mathrm{msec}$ after the prompt.

Stimulus presentation in the fixation condition was made similar to that in the vergence condition. In the convergence experiment fixation condition the subject maintained fixation on the far target throughout each test interval. The decremental stimulus was delivered $0,200,400$, or $600 \mathrm{msec}$ after the prompt. When vision was tested in the divergence experiment, the subject maintained fixation on the near target on fixation trials and diverged the eyes from the near to the far target on vergence trials.

\section{Data collection and analysis}

A storage oscilloscope was used to monitor the 2 successive eye movements and the stimulus presentation in each trial. In the vergence condition, data were rejected if the stimulus failed to occur at the appropriate time in relation to the eye movement, if the EOG showed any evidence of a saccade or other departure from the usual vergence waveform, or if the digital clock failed to record. In the fixation condition, data were rejected if the EOG indicated the occurrence of eye movement.

Trials were grouped into blocks of vergence or fixation trials of similar time delay. Each block started with practice trials in which a decremental stimulus of high amplitude was used to familiarize the subject with the nature and timing of the task. Then, the amplitude of stimulus decre- ment was reduced to one of the steps within the lower range used for actual testing and was varied every 5-10 trials. Stimulus amplitude and delay were counterbalanced within and across sessions. The subject took a break whenever desired. Each experimental session normally lasted 1-1.5 hr. Subjects required 12-14 sessions to complete the experiment.

The subject viewed a range of values of stimulus decrement within each experimental condition, from easily seen to imperceptible. Each judgment was associated with a specific amplitude $(\Delta I)$ of stimulus decrement and a specific value of stimulus delay $(\Delta t)$. For analysis, data in the movement condition were grouped into 60 -msec-wide time bins according to the value of $\Delta t$. From 14-40 (mean, 20) judgments were obtained from a subject at each value of $\Delta I$ that was included within each time bin. The proportions of correct judgments were found, converted to $Z$ scores, and plotted as a function of $\log (\Delta I / I)$.

The method of least squares was used to fit regression lines to these data. From 3-6 data points were used to obtain each fit. In the end, from 60-120 judgments were incorporated into a psychometric function for each time bin. The data included the proportion associated with the largest value of stimulus decrement at which the subject's judgments were $50 \%$ correct, up to the smallest value of stimulus decrement at which the subject's judgments were $100 \%$ correct. For the $Z$-score conversion, any percentage less than chance $(50 \%)$ was arbitrarily assigned a value of $50 \%$ and a perfect score $(100 \%)$ was assigned a value of halfway down to the next possible percentage point. Threshold was defined as that value of stimulus decrement associated with $75 \%$ correct judgments as interpolated from the fitted curve. In order to test for any possible effects of varying the delay setting, visual sensitivity data from the fixation condition were analyzed according to delay setting. Since there was no effect of varying delay settings alone (sce Rcsults), these data were then reanalyzed en masse. The measure of suppression was the difference between threshold values of $\log (\Delta I / I)$ in the vergence and the fixation condition.

\section{Subjects}

Four adult, human subjects participated in these experiments. J.P.K. was a relatively inexperienced observer compared to the other subjects. All were given preliminary practice and had vergence movements that were symmetrical and uncontaminated by saccades. All had normal vision without correction or with slight correction. S.B.S. wore lightly tinted contact lenses. Subjects were not informed of the progress of the experiment during testing.

\section{Results}

\section{Fixation condition}

There is no effect of changing the delay of the stimulus in the fixation condition (Fig. 3). When the proportions of correct judgments are plotted as a function of the amplitude of stimulus decrement for the 4 delay settings, it is clear that when no movement occurs, variation in stimulus delay produces no systematic effect within or across subjects. Therefore, these data were collapsed across delay settings to produce a single psychometric function for each subject from which a single threshold value of $\log (\Delta I / I)$ was obtained.

\section{Time course of suppression during convergence}

A similar loss of sensitivity was found during convergencc in all subjects. In Figure 4, sensitivity, the reciprocal of the threshold value of $(\Delta I / I)$, is plotted on a log scale as a function of the time of presentation of the stimulus in relation to time of onset of the subject's convergence as signaled by the trigger in the EOG circuit. The horizontal bar indicates the average interval of the subjects' convergence movement, based on photography. Sensitivity in the fixation condition is shown on the left of the figure. A loss in sensitivity from fixating levels is measured when stimuli are presented just before the eyes start to converge and is greatest when stimuli are presented around the time of detection of vergence by the EOG trigger, the point of maximum eye acceleration. If the data are replotted with respect to the time of movement onset, the curves shift along the abscissa to different points after vergence initiation so that they are no 


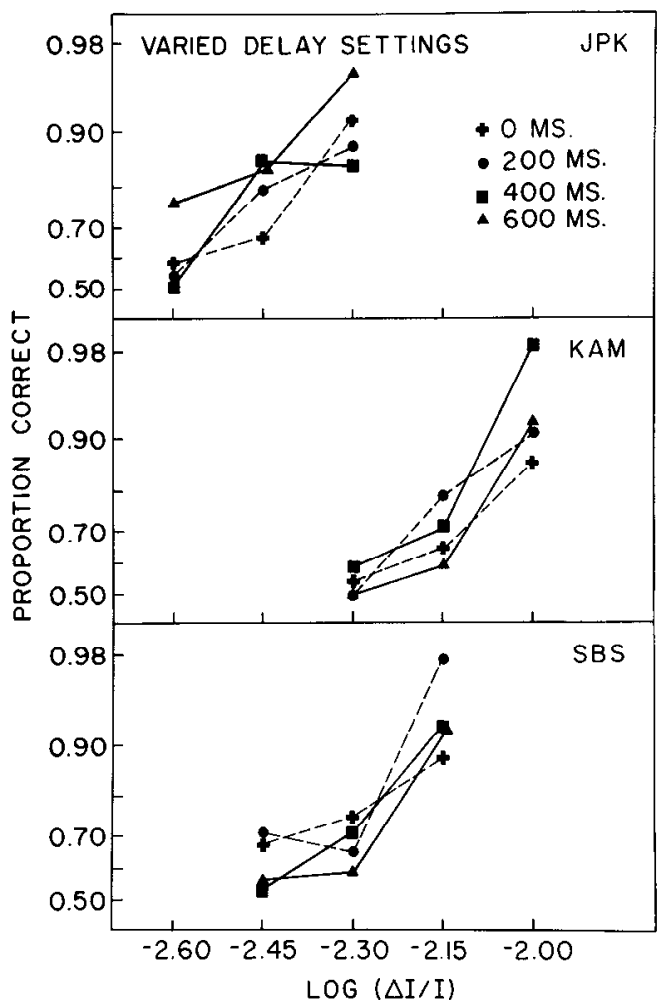

Figure 3. Varied time of stimulus presentation in the fixation condition. For each of three subjects the proportion of correct judgments is plotted as a function of the value of stimulus decrement for time delays of $0,200,400$, and $600 \mathrm{msec}$ following the prompt when no eye movement occurred.

longer regularly aligned with one another. This suggests that the time of loss in sensitivity is more closely associated with the time of maximum eye acceleration than the time of initial eye rotation. The greatest difference in sensitivity between the vergence and the fixation condition equals $0.44,0.44$, and $0.41 \mathrm{log}$ units for J.P.K., K.A.M., and S.B.S., respectively. From this peak of suppression, sensitivity returns to premovement levels over a less rapid and more varied time course resembling that seen during saccadic suppression (e.g., Volkmann and Moore, 1978).

\section{Suppression with mydriasis and cycloplegia}

Changes in pupil diameter and in the state of accommodation accompany vergence and can affect vision (Riggs, 1965). The change in sensitivity mcasurcd during convergence was practically the opposite of what one would predict based on the change in pupil diameter measured during convergence. Also, the adjustments or errors in accommodation in this experiment seem unlikely to affect detection of the diffuse decrements of Ganzfeld illumination. Nevertheless, to eliminate these possible influences on the experimental results, vergence sensitivity was examined with the subject's pupil diameter and accommodative state fixed.

Data were collected following repeated application of $0.5 \%$ tropicamide (Mydriacyl, Alcon) eye drops to induce mydriasis and cycloplegia. The full effects of the drug occurred relatively promptly, within 15 to $30 \mathrm{~min}$, and were maintained during testing. The subject's pupils were fully dilated, fixed at $8.5 \mathrm{~mm}$ o.u., and unreactive to bright light. Near vision was blurred. Visual acuity (in Snellen notation) measured binocularly with a Bausch \& Lomb Ortho-Rater before administration of eye drops was $20 / 18$ for near vision and $20 / 15$ for far vision. Following drug administration, near vision decreased to $20 / 33$, while far vision remained normal, 20/14.

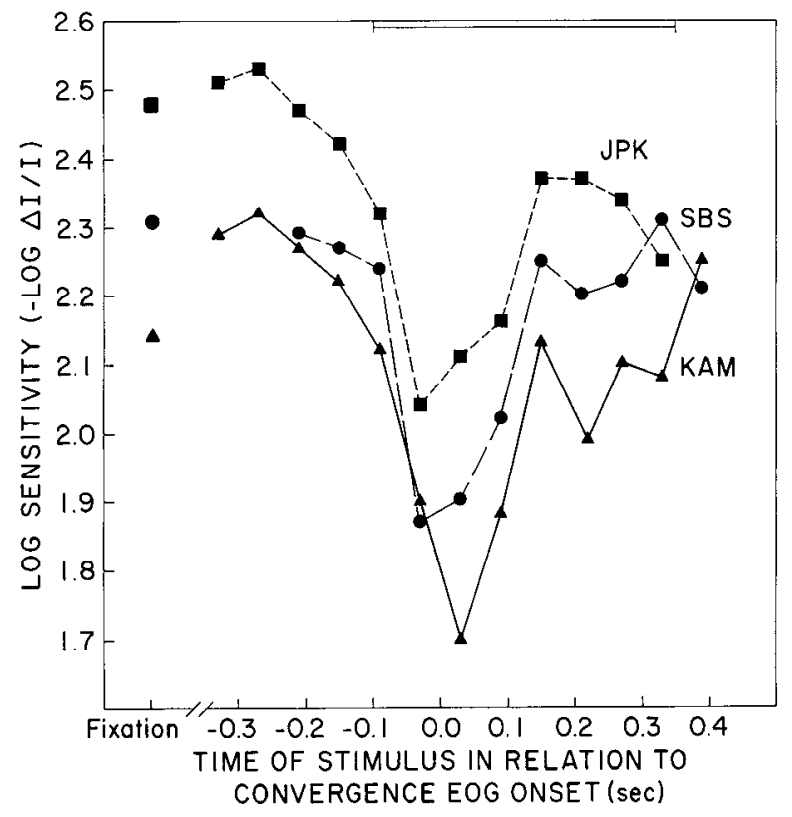

Figure 4. Visual sensitivity before, during, and following convergence. Log sensitivity, the reciprocal of threshold, is plotted as a function of the time of stimulus presentation with respect to onset of convergence as signaled by a trigger in the EOG circuit. Sensitivity in the fixation condition collapsed across time delays is indicated at left. The average time of occurrence of convergence determined photographically is indicated by the bar across the top of the figure.

The basic psychophysical procedure and viewing conditions were identical to those used in the first experiment, except that the decremental stimulus was presented just when the trigger detected convergence, near the point of maximal suppression. In the fixation condition, the stimulus was presented immediately following the prompt. To obtain 40-50 judgments for each value of stimulus decrement required 3 experimental sessions, 1 pcr day.

The results in Figure 5 show that visual sensitivity during convergence decreases when pupil diameter and the state of accommodation are held constant. The subject's proportion of correct judgments is plotted for each value of stimulus decrement with the lines of best fit for the 2 conditions. The convergence and fixation functions do not overlap and the difference

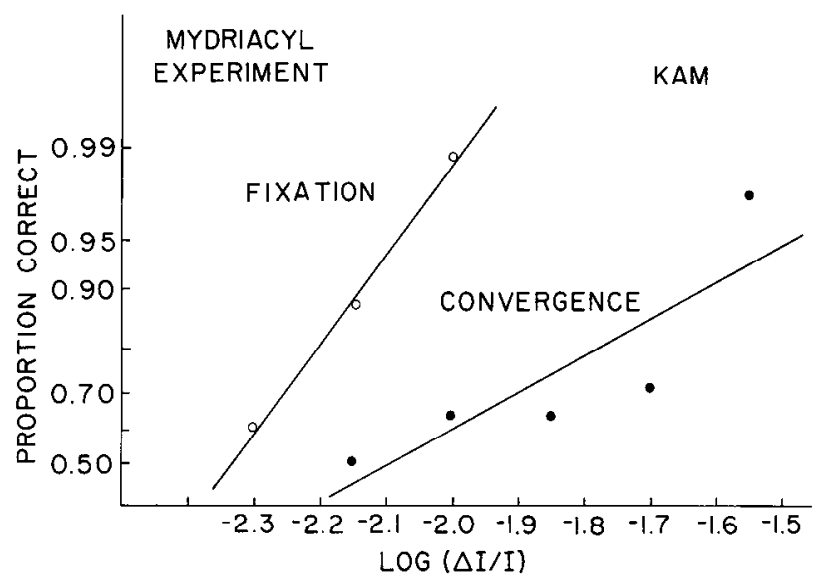

Figure 5. Convergence-related suppression during cycloplegia and dilatation. The proportion of correct judgments at each level of stimulus decrement tested and best-fitted lines are shown for the convergence and fixation conditions. Subject K.A.M. was tested when her state of accommodation and pupil diameter were fixed. 


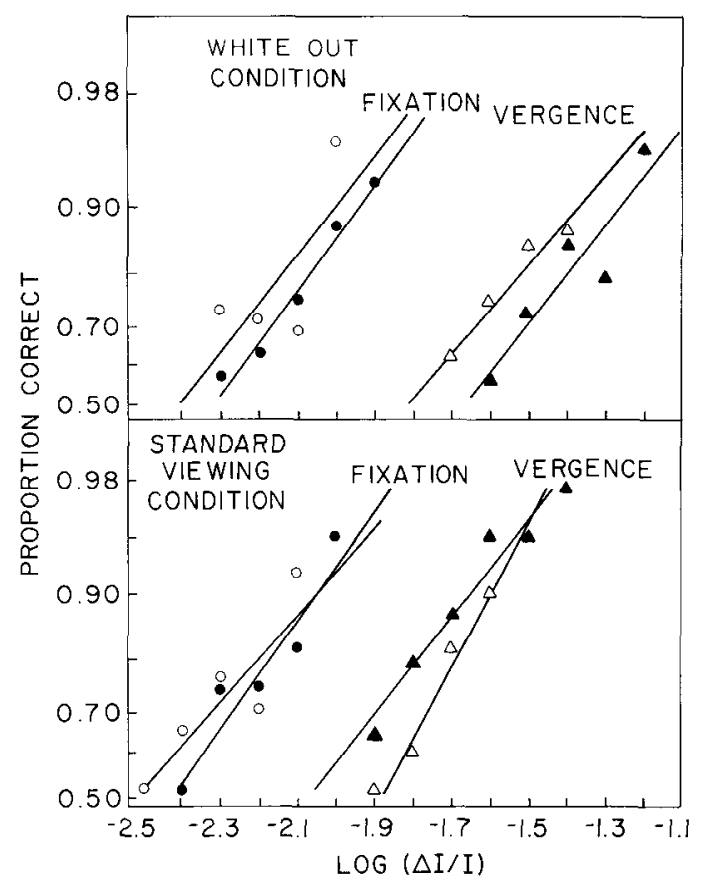

Figure 6. Convergence- and divergence-related suppression during whiteout. The proportion of correct judgments at each level of stimulus decrement tested and best-fitted lines are shown for subject R.K.M. during convergence $(\Delta)$ and divergence $(\Delta)$ and for far $(O)$ and near $(\bullet)$ fixation conditions. Whiteout data are shown above and standard viewing condition data are shown below.

in threshold sensitivity between the two conditions equals 0.39 log unit. Since this subject showed similar threshold values without mydriasis and cycloplegia (Fig. 4), these results demonstrate that change in pupil size and in accommodation cannot explain convergence-related suppression.

\section{Suppression during whiteout}

To eliminate the possibility that the loss in sensitivity during vergence was caused by smear or masking, suppression was measured in a completely homogeneous visual environment. To produce the whiteout condition, the subject wore translucent white, plastic, diffusing goggles over each eye (Riggs and Manning, 1982). The diffusers were shaped to fit closely around the orbit, eliminating any gap between the skin and the plastic. With the subject's head positioned in the Ganzfeld, the diffusers were illuminated by the light reflected from the Ganzfeld surface, which gave a practically uniform luminance over the entire visual field. This effectively removed all contours, including the outline of the nose and cheeks.

Vision was tested in both the whitcout condition and in the standard viewing condition. To provide equality of luminance for the 2 conditions, lattice lamp covers applied to the fluorescent lamps in the standard viewing condition were removed in the whiteout condition (when the observer wore the goggles). The luminance of the field in the whiteout condition, 10.1 footLamberts, was estimated by placing a diffuser securely over the light-sensitive portion of the photometer while taking measurements. Luminance in the standard viewing condition was 9.8 foot-Lamberts.

The vergence EOG was used to coach the subject, R.K.M., to make vergence movements of similar amplitude, approximately $7.7^{\circ}$, in both conditions. R.K.M. made smooth, symmetrical vergence movements during whiteout, and his vergence EOG looked similar, with or without diffusers. The amplitude of R.K.M.'s vergence increased slightly over the course of testing.

The psychophysical procedure was similar to that used in the above experiment. Vision was tested during both convergence and divergence (in alternate sessions), under standard viewing conditions and during whiteout (within the same session). To collect 40-45 judgments at cach level of stimulus decrement in the 8 conditions required 12 sessions.

The results indicate that visual suppression during convergence and divergence persists, undiminished, under whiteout conditions. The subject's percentage of correct judgments is plotted at each value of stimulus decrement (Fig. 6) for the whiteout and the standard viewing conditions with lines of best fit. Regardless of the viewing condition, the subject is less sensitive during vergence than during fixation and exhibits just as much suppression while wearing diffusing goggles as in the standard viewing condition. Suppression associated with convergence equals $0.54 \log$ unit in the standard viewing condition and $0.61 \mathrm{log}$ unit in the whiteout condition. Suppression associated with divergence equaled $0.39 \mathrm{log}$ unit in the standard viewing condition and $0.67 \mathrm{log}$ unit in the whiteout condition. There is no significant difference in the magnitude of suppression across the different conditions (Pearson $\chi^{2} ; d f=1, \chi^{2}$ obs $=$ 2.678 , n.s.). These results demonstrate that convergence- and divergence-related suppression persists even during whiteout.

\section{Discussion}

A roughly $0.5 \log$ unit loss in visual sensitivity is present when vision is tested near the onset of convergence or divergence in which each eye moves through $2^{\circ}-3^{\circ}$ (Manning and Riggs, 1984) or $7^{\circ}-9^{\circ}$ (in the present study). The loss of visual sensitivity measured during vergence in these experiments cannot be attributed to retinal masking, or retinal image smear, since suppression is still present under conditions of whiteout. Furthermore, suppression persists when the observer's pupil and state of accommodation are fixed, so the loss in sensitivity cannot be attributed to these ancillary processes that accompany vergence. The relatively small rotational inertia involved in the slow, gliding vergence movement renders unlikely a loss in sensitivity due to retinal shearing. Instead, the results support the hypothesis that vision in these experiments is attenuated through more centrally originating neural inhibition that occurs as a corollary to initiation of vergence.

The suppression of vision measured during convergence and divergence is quantitatively similar to that associated with saccades. Moreover, the time course of suppression during convergence bears a striking resemblance to the time course of both saccadic and blink-related suppression (e.g., Manning et al., 1983b; Volkmann, 1976). During saccade- and vergence-related suppression tested with brief flashes, the period of impaired vision lasts $300-400 \mathrm{msec}$, most affects test stimuli presented at the onset of the movement, and begins somewhat more abruptly than it ends. It is interesting to note that the peak of suppression is found when test stimuli are presented close to the time that the EOG detects the movement, that is, when eyc acceleration is near maximum. Because the duration, magnitude, and time course of the change in visual sensitivity during saccades and vergence are similar, despite the great differences in the nature of the 2 movements, it is unlikely that the momentary amplitude of the suppression could be determined by the dynamic properties of the eye movement. Again, one is led to the supposition that the duration and the amplitude of suppression are centrally controlled.

Visual suppression is associated with commonly occurring eye and lid movements that could interrupt or interfere with perception. The potentially disruptive perceptual consequences of a saccade, which in effect sends the visual field streaking across the retina, or a blink, which obscures light and contour for 40200 msec (Doane, 1980; Moses, 1975; Ponder and Kennedy, 1928) are dramatic. But vergence, too, may produce disruptive percepts. If a haploscope is used to give equal and opposite motions to the retinal images, or if one pushes simultaneously 
near the outer canthus of the 2 eyes, the world appears to move. Further, no diplopia follows changes in fixation between objects of different depths in space, even though sometimes nearly a second is required before convergence approaches the required level (Westheimer and Mitchell, 1956). So, as is true for saccades (Helmholtz, 1954), we must compensate in some manner for the sensory effects of actively produced vergence.

However, if a corollary signal acts on visual neurons during eye movements, it is likely to be attenuating visual sensitivity, rather than compensating in some other manner. The effects of suppression appear to be greater near threshold, rather than suprathreshold (blinks: Riggs et al., 1981; saccades: Riggs et al., 1982), and it is difficult to see how a threshold change in sensitivity might be directly involved in compensating for many of the sensory effects of an eye movement that are measurable above detection threshold (e.g., White et al., 1980). Also, the occurrence of visual suppression and the maintenance of perceptual stability are not necessarily linked (Nagle et al., 1980). Instead, visual suppression may be an indication of reduced sensitivity or activity of visual neurons that results from an oculomotor command.

Direct evidence of this inhibitory signal remains to be found (although see Buisseret and Maffei, 1983; Letelier et al., 1985); however, evidence for other types of corollary signals exists in the primate oculomotor literature (e.g., Guthrie et al., 1983). Furthermore, there are striking examples in sensory systems of many species of corollary signals to motor commands that act to reduce an animal's sensitivity to the sensory consequences of its own motor activity. Stimulation of the lateral line organ in fish and frogs by the ripples in the water generated through swimming movements is suppressed by an efferent discharge produced as a corollary to movement (Russell, 1976). In these animals, bursts of impulses from efferent fibers impinge directly onto receptors in the lateral line and sensitivity is reduced by $1-2 \log$ units depending on how vigorously the animal swims. A similar control mechanism is present in the auditory system of bats (Suga and Schlegal, 1972). When the bat's muscles of vocalization discharge, they simultaneously cause its ear muscles to contract, impeding incoming soundwaves. Further attenuation occurs through neural mechanisms in central auditory pathways. In one species of electric fish, an efferent signal is produccd as a corollary to the motor command that discharges the electric organ (Bell, 1981). The corollary signal appears to reduce the central neural effects on the animal's receptors that would be evoked by its electric organ discharge.

There are also impressive examples of such signals in the oculomotor system of nonhuman species. In the toad, the offresponse of 1 class of retinal ganglion cells fails to occur when the animal closes its eyelids (Borchers and Ewert, 1978; Llinás and Precht, 1976). This lack of response appears to result from an inhibitory, efferent signal that accompanies the command to lower the lids. In the locust, the response of a central, visual interneuron is reduced by nearly $1 \log$ unit by an efferent corollary discharge that accompanies a "saccade," which is actually a head turn that occurs at saccadic angular velocities (Heitler, 1983; Zaretsky, 1982). Finally, visual neurons in cortical area 17 of the cat are inhibited at the time of an eyeblink, even though the eyelid does not block the pupil (Buisseret and Maffei, 1983), which the authors attribute to neural inhibition associated with a blink.

The reduction of vision measured during vergence in the present experiment may be one example of a more generally occurring phenomenon of sensory decrement with movement. It is now well established that somatosensory signals at several levels of the afferent pathway are attenuated through centrifugal control during voluntary limb movement in animals and man (Angel et al., 1985; Coulter, 1974; Ghez and Pisa, 1972; Rushton et al., 1981; Starr and Cohen, 1985; Tsumoto et al., 1975). Modulation of somatosensory information during limb move- ment appears to parallel, in many ways, the modulation of visual information during eye movement. By diminishing sensations likely to result from the self-produced movement, sensitivity to more important external events could be maintained. These examples suggest ways in which an oculomotor signal could function in humans to produce a suppression of vision during vergence and, more generally, during eye movements.

It is now clear that visual suppression affects vision during almost every type of eye-related movement. Only during a smooth pursuit movement has suppression not been found (Starr et al., 1969). However, vision has never been tested near the start of such a movement. This means that during most eye movements under normal viewing conditions, vision is reduced in at least one manner, but is more commonly reduced through the combined effects of several sources. When visual masking and image smear also occur during saccades, the effects of suppression may, in comparison, seem subtle. However, visual suppression appears to accompany all saccades, blinks, and vergence movements, regardless of whether masking is present, so suppression is therefore a more generally occurring phenomenon than masking.

\section{References}

Angel, R. W., M. Weinrich, and D. Siegler (1985) Gating of somatosensory perception following movement. Exp. Neurol. 90: 395-400.

Alpern, M. (1953) Metacontrast. J. Opt. Soc. Am. 29: 631-646.

Armington, J. C. (1981) Visually evoked cortical potentials accompanying blinks. Invest. Ophthalmol. Vis. Sci. 20: 691-695.

Bahill, A. T., and L. Stark (1979) Trajectories of saccadic eye movements. Sci. Am. 240: 84-93.

Bell, C. C. (1981) An efference copy which is modified by reafferent input. Scicnce 214: 450-453.

Borchers, H. W., and J. P. Ewert (1978) Eye closure in toads (Bufo $b u f o L$.) does not produce off responses in retinal on-off ganglion cells: A question of efferent commands. J. Comp. Physiol. 125: 301-308.

Breitmeyer, B. G., and L. Ganz (1976) Implication of sustained and transient channels for theories of visual pattern masking, saccadic suppression and information processing. Psychol. Rev. 83: 1-36.

Brooks, B. A., and A. F. Fuchs (1975) Influence of stimulus parameters on visual sensitivity during saccadic eye movement. Vision Res. 15: 1389-1398

Buisseret, P., and L. Maffei (1983) Suppression of visual cortical activity following tactile periorbital stimulation: Its role during eyeblinks. Exp. Brain Res. 151: 463-466.

Campbell, F. W., and R. H. Wurtz (1978) Saccadic omission: Why we do not see a grey-out during a saccadic eye movement. Vision Res. 18: 1297-1303.

Collewijn, H. (1969) Changes in visual evoked responses during the fast phase of optokinetic nystagmus in rabbit. Vision Res. 9: 803814.

Coulter, J. D. (1974) Sensory transmission through lemniscal pathway during voluntary movement in the cat. J. Neurophysiol. 37: 831845.

Doane, M. G. (1980) Interaction of eyelids and tears in corneal wetting and the dynamics of the normal human eyeblink. Am. J. Ophthalmol. 89: $507-516$

Dodge, R. (1900) Visual perception during eye movement. Psychol. Rev. 7: 454-465.

Dodge, R. (1905) The illusion of clear vision during eye movement. Psychol. Bull. 2: 193-199.

Duffy, F., and C. Lombroso (1968) Electrophysiological evidence for visual suppression prior to the onset of a voluntary saccadic eye movement. Nature 218: 1074-1075.

Enright, J. T. (1984) Saccadic anomalies: Vergence induces large departures from ball-and-socket behavior. Vision Res. 24: 301-308.

Ghez, C., and M. Pisa (1972) Inhibition of afferent transmission in cuneate nucleus during voluntary movement in the cat. Brain Res. 40: $145-151$.

Gregory, R. L. (1966) Eye and Brain, pp. 91-115, McGraw-Hill, New York.

Grehn, F., O.-J. Grusser, and D. Stange (1984) Effect of short-term intraocular pressure increase on cat retinal ganglion cell activity. Behav. Brain Res. 14: 109-121. 
Gross, E., H. Vaughan, and E. Valenstein (1967) Inhibition of visual evoked responses to patterned stimuli during voluntary eye movements. Electroencephalogr. Clin. Neurophysiol. 22: 204-209.

Guthrie, B. L., J. D. Porter, and D. L. Sparks (1983) Corollary discharge provides accurate eye position information to the oculomotor system. Science 221: 1193-1195.

Heitler, W. J. (1983) Suppression of a locust visual interneurone (DCMD) during defensive kicking. J. Exp. Biol. 104: 203-215.

Helmholtz, H. von (1954) Handbuch der Physiologischen Optik, Vol. 3. (Translated from the 3rd German Ed. by J. P. C. Southall, 1910), pp. 242-247, Optical Society of America, Menasha, WI.

Holt, E. B. (1903) Eye movement and central anaesthesia. Psychol. Rev. 4: 1-45.

Keller, E. L., and D. A. Robinson (1972) Abducens unit behavior in the monkey during vergence movements. Vision Res. 12: 369-382.

Latour, P. L. (1962) Visual threshold during eye movements. Vision Res. 2: 261-262.

Lefton, L. (1972) Metacontrast: A review. Psychonom. Monogr. Suppl. 4: $245-255$.

Letelier, J. C., J. Wallman, and G. Marin (1985) Centrifugal fibers of the optic nerve carry eye movement signals. Soc. Neurosci. Abstr. 11: 1043 .

Llinás, R., and W. Precht (1976) Frog Neurobiology: A Handbook, Springer, Berlin.

MacKay, D. M. (1973) Visual stability and voluntary eye movements. In Handbook of Sensory Physiology, Vol. VII/3, Central Visual Information A, R. Jung, ed., pp. 307-331, Springer, Berlin.

Manning, K. A., and L. A. Riggs (1984) Vergence eye movements and visual suppression. Vision Res. 24: 521-526.

Manning, K. A., L. A. Riggs, and J. K. Frost (1983a) Visual suppression during spontaneous eyeblinks. Invest. Ophthalmol. Vis. Sci. (Suppl.) 24: 187.

Manning, K. A., L. A. Riggs, and J. K. Komenda (1983b) Reflex eyeblinks and visual suppression. Percept. Psychophys. 34: 250-256.

Matin, E. (1974) Saccadic suppression: A review and an analysis. Psychol. Bull. 81: 899-917.

Mays, L. E. (1983) Neurophysiological correlates of vergence eye movements. In Vergence Eye Movements, C. M. Schor and K. J. Cuiffreda, eds., Butterworths, Boston.

Mays, L. E. (1984) Neural control of vergence eye movements: Convergence and divergence neurons in midbrain. J. Neurophysiol. 51: 1091-1108.

Mays, L. E., and J. D. Porter (1984) Activity of midbrain neurons that encode vergence velocity. Soc. Neurosci. Abstr. 9: 1086.

Mitrani, L., S. T. Mateef, and N. Yakimoff (1970) Smearing of the retinal image during voluntary saccadic eye movements. Vision Res. 10: 405-409.

Mollon, J. D., and P. G. Polden (1978) On the time constants of tachistoscopes. Q. J. Exp. Psychol. 30: 555-567.

Moses, R. A. (1975) The eyelids. In Adler's Physiology of the Eye, 6th ed., R. A. Moses, ed., pp. 4-13, Mosby, St. Louis.

Nagle, M., B. Bridgeman, and L. Stark (1980) Voluntary nystagmus, saccadic suppression, and stabilization of the visual world. Vision Res. 20: 717-721.

Pearce, D. G., and E. Porter (1970) Changes in visual sensitivity associated with voluntary saccades. Psychonom. Soc. 19: 225-227.

Ponder, E., and Kennedy, W. P. (1928) On the act of blinking. Q. J. Exp. Psychol. 18: 89-1 10.

Richards, W. (1968) Visual suppression during passive eye movement. J. Opt. Soc. Am. 58: 1159-1160.

Richards, W. (1969) Saccadic suppression. J. Opt. Soc. Am. 59: 617624.

Riggs, L. A. (1965) Visual acuity. In Vision and Visual Perception. C. H. Graham, ed., pp. 333-334, Wiley, New York.

Riggs, L. A., and K. A. Manning (1982) Saccadic suppression under conditions of whiteout. Invest. Ophthalmol. Vis. Sci. 23: 138-143.

Riggs, L. A., P. A. Merton, and H. Morton (1974) Suppression of visual phosphenes during saccadic eye movements. Vision Res. 14: 997-1010.

Riggs, L. A., F. C. Volkmann, and R. K. Moore (1981) Suppression of the blackout due to blinks. Vision Res. 21: 1075-1079.

Riggs, L. A., F. C. Volkmann, R. K. Moore, and A. G. Ellicott (1982) Perception of suprathreshold stimuli during saccadic eye movement. Vision Res. 22: 423-428.

Rushton, D. N., J. C. Rothwell, and M. C. Craggs (1981) Gating of somatosensory evoked potentials during different kinds of movements in man. Brain 104: 465-491.
Russell, I. J. (1976) Amphibian lateral line receptors. In Frog Neurobiology: A Handbook, R. Llinás and W. Precht, eds., pp. 514-550, Springer, Berlin.

Sperry, R. W. (1950) Neural basis of the spontaneous optokinetic response produced by visual inversion. J. Comp. Physiol. Psychol. 43: 482-489.

Starr, A., and L. G. Cohen (1985) "Gating" of somatosensory evoked potentials begins before the onset of voluntary movement in man. Brain Res. 348: 183-186.

Starr, A., R. Angel, and H. Yeates (1969) Visual suppression during smooth following and saccadic eye movements. Vision Res. 9: 195197.

Suga, N., and P. Schlegal (1972) Neural attenuation of responses to emitted sounds in echolocating bats. Science 177: 82-84.

Tsumoto, T., S. Nakamura, and K. Iwama (1975) Pyramidal tract control over cutaneous and kinesthetic sensory transmission in the cat thalamus. Exp. Brain Res. 22: 281-294.

Volkmann, F. C. (1962) Vision during voluntary saccadic eye movements. J. Opt. Soc. Am. 52: 571-578.

Volkmann, F. C. (1976) Saccadic suppression: A brief review. In Eye Movements and Psychological Processes, R. A. Monty and J. W. Senders, eds., pp. 73-154, Lawrence Erlbaum, Hillsdale, NJ.

Volkmann, F. C., and R. K. Moore (1978) Saccadic eye movements and the perception of a clear and continuous visual world. In Visual Psychophysics and Physiology, J. C. Armington, J. Krauskopf, and B. R. Wooten, eds., pp. 353-362, Academic, New York.

Volkmann, F. C., A. M. L. Schick, and L. A. Riggs (1968) Time course of visual inhibition during voluntary saccades. J. Opt. Soc. Am. 58 . 562-569.

Volkmann, F. C., L. A. Riggs, R. K. Moore, and K. D. White (1978a) Central and peripheral determinants of saccadic suppression. In Eye Movements and the Higher Psychological Functions, J. W. Senders, D. A. Fisher, and R. A. Monty, eds., Lawrence Erlbaum, Hillsdale, NJ

Volkmann, F. C., L. A. Riggs, K. D. White, and R. K. Moore (1978b) Contrast sensitivity during saccadic eye movements. Vision Res. 18: 1193-1199.

Volkmann, F. C., L. A. Riggs, and R. K. Moore (1979) A comparison of saccades and blinks in suppression of vision. Invest. Ophthalmol. Vis. Sci. (Suppl.) 18: 140.

Volkmann, F. C., L. A. Riggs, and R. K. Moore (1980) Eyeblinks and visual suppression. Science 207: 900-902.

Volkmann, F. C., L. A. Riggs, A. G. Ellicott, and R. K. Moore (1982) Measurements of visual suppression during opening, closing, and blinking of the eyes. Vision Res. 22: 991-996.

von Holst, E. (1954) Relations between the central nervous system and the peripheral organs. Br. J. Anim. Behav. 2: 89-94.

Weisstein, N. (1972) Metacontrast. In Handbook of Sensory Physiology, Vol. VII/4, Visual Psychophysics, D. Jameson and L. M. Hurvich, eds., pp. 233-244, Springer, Berlin.

Westheimer, G., and A. M. Mitchell (1956) Eye movement responses to convergence stimuli. Arch. Ophthalmol. 55: 848-856.

White, K. D., R. B. Post, and H. W. Leibowitz (1980) Saccadic eye movements and body sway. Science 208: 621-623.

White, K. D., J. H. Krantz, R. K. Moore, and K. A. Manning (1984) Blink-related evoked potentials for incremental vs. decremental test pulses of light. Invest. Ophthalmol. Vis. Sci. (Suppl.) 25: 297.

Wibbenmeyer, R., J. A. Stern, and S. C. Chen (1983) Elevation of visual threshold associated with eyeblink onset. Intern. J. Neurosci. 18: 279-286.

Woodworth, R. (1906) Vision and localization during eye movements. Psychol. Bull. (Suppl.) 3: 68-70.

Woodworth, R. (1938) Experimental Psychology, Holt, New York.

Zaretsky, M. (1982) Quantitative measurements of centrally and retinally generated saccadic suppression in a locust movement detector neuron. J. Physiol. (Lond.) 328: 521-533.

Zuber, B., and L. Stark (1966) Saccadic suppression: Elevation of visual threshold associated with saccadic eye movements. Exp. Neurol. 16: 65-79.

Zuber, B., A. Crider, and L. Stark (1964) Saccadic suppression associated with microsaccades. Q. Prog. Rep. Res. Lab. Elect. M.I.T. 74: 244-249.

Zuber, B., L. Stark, and M. Lorber (1966) Saccadic suppression of the pupillary light reflex. Exp. Neurol. 14: 351-370. 